\title{
ESPÉCIES DE CURCULIONIDAE (INSECTA, COLEOPTERA) EM CULTURA DE COQUEIRO ANÃO VERDE, EM LINHARES, ES, BRASIL
}

\author{
Emerson Fraga Comério ${ }^{1} \&$ Vera Lúcia Rodrigues Machado Benassi ${ }^{2}$ \\ ${ }^{1}$ Secretaria Municipal de Meio Ambiente e Recursos Hídricos Naturais, Av. Comendador Rafael, 1743, CEP 29900-056, \\ Linhares, ES, Brasil. E-mail: emersoncomerio@hotmail.com \\ ${ }^{2}$ Instituto Capixaba de Pesquisa, Assistência Técnica e Extensão Rural, Laboratório de Controle Biológico, Rod. BR 101, Km \\ 151, Bairro Bebedouro, Linhares, ES, Brasil.
}

\section{RESUMO}

O objetivo deste estudo foi determinar as espécies de curculionídeos que ocorrem associadas a um cultivo de coqueiro anão verde, Cocos nucifera L., no município de Linhares, ES. Para a amostragem dos insetos foram utilizadas armadilhas amarelas tipo Moericke. O levantamento foi realizado semanalmente, no período de março/2008 a fevereiro/2009. Foi coletado um total de 952 exemplares pertencentes a 11 espécies: Parisoschoenus obesulus (409 exemplares), Andranthobius bondari (293), Metamasius hemipterus (88), Apion sp. (65), Baris sp. (41), Sibariops sp. (14), Conotrachelus sp. (13), Auletobius sp. (13), Sithopilus zeamais (10), Sibinia sp. (3) e Anthonomus sp. (3). Destas, somente as espécies P. obesulus, A. bondari e M. hemipterus são registradas na literatura associadas ao coqueiro anão verde.

Palavras-chave: Cocos nucifera, curculionídeos, levantamento

\section{SPECIES OF CURCULIONIDAE (INSECTA, COLEOPTERA) IN CROP OF THE GREEN DWARF COCONUT PALM, IN LINHARES, STATE OF ESPÍRITO SANTO, BRAZIL}

\begin{abstract}
The aim of this study was to determine the species of weevils that occur associated with a crop of green dwarf coconut palm, Cocos nucifera L., in Linhares, ES. Yellow traps Moericke type were used for sampling the insects. The survey was conducted weekly, from March/2008 to February/2009. A total of 952 specimens belonging to 11 species were collected: Parisoschoenus obesulus (409 exemplars), Andranthobius bondari (293), Metamasius hemipterus (88), Apion sp. (65), Baris sp. (41), Sibariops sp. (14), Conotrachelus sp. (13), Auletobius sp. (13), Sithopilus zeamais (10), Sibinia sp. (3) and Anthonomus sp. (3). From these, only the species P. obesulus, A. bondari and $M$. hemipterus are reported in the literature associated with the green dwarf coconut palm.
\end{abstract}

Keywords: Cocos nunifera, weevils, survey

\section{INTRODUÇÃO}

O coqueiro, Cocos nucifera $\mathrm{L}$. (Arecaceae), é uma planta tipicamente tropical, originada do Sudeste Asiático e encontra-se disseminada em diversas regiões do globo (Ferreira et al., 1997). O Brasil possui cerca de 280 mil hectares cultivados com coqueiro, distribuídos, praticamente, em quase todo o território nacional com 
produção equivalente a dois bilhões de frutos (FAO, 2011). Em termos de produtividade, os estados do Rio de Janeiro, Espírito Santo e Pará figuram entre aqueles com maior rendimento no cenário nacional, com um aumento significativo na maior produção de frutos por hectare, principalmente devido à variedade Anã Verde que apresenta maior aplicabilidade no uso industrial e pela maior quantidade e qualidade de água de coco em relação às outras variedades (Martins \& Jesus Júnior, 2011).

Devido à importância da cultura do coqueiro para o Espírito Santo, o manejo fitossanitário é de extrema importância, uma vez que as pragas deste cultivo representam problemas limitantes à produção, pois atacam os diferentes estágios de seu crescimento e desenvolvimento, causando danos às diferentes partes da planta $\mathrm{e}$ respondendo de maneira significativa pelo depauperamento do cultivo e baixa produtividade (Ferreira et al., 1998).

Estudos mais recentes relatam a ocorrência de vinte e quatro pragas consideradas principais para a espécie $C$. nucifera, com destaque para as espécies de coleópteros da família Curculionidae, Ameris ynca (Sahlberg, 1823), conhecido como "broca-da-ráquis-foliar", Homalinotus coriaceus (Gyllenhal, 1836), "broca-dopedúnculo-floral"; Parisoschoenus obesulus Casey 1922, "gorgulho-dos-frutos-e-flores", Rhinostomus barbirostris Fabricius, 1775, "broca-do-estipe-do-coqueiro"

Rhynchophorus palmarum Linnaeus, 1764, "broca-do-olho-do-coqueiro" (Ferreira et al., 1998).

Apesar da importância dos curculionídeos ao coqueiro, não há informações na literatura sobre o monitoramento e levantamento de espécies que ocorrem associadas ao coqueiro anão verde nos municípios produtores do Estado do Espírito Santo. A posse de informações é de grande importância, visto que, sabendo quais espécies e em que períodos elas ocorrem em maior abundância, é possível planejar o seu manejo e controle.

Considerando a escassez de tais informações, este trabalho teve como objetivo levantar as espécies de curculionídeos que ocorrem associadas a um cultivo de coqueiro anão verde.

\section{MATERIAL E MÉTODOS}

O estudo foi realizado em uma lavoura de cinco hectares, cultivada com $C$. nucifera cv. Anã Verde, plantas de nove anos de idade, localizada na Fazenda Experimental do Instituto Capixaba de Pesquisa, Assistência Técnica e Extensão Rural (INCAPER) (19 ${ }^{\circ} 25^{\prime} 6^{\prime \prime} \mathrm{S} ; 40^{\circ} 4^{\prime} 30^{\prime \prime}$ W), em Linhares, Estado do Espírito Santo.

Os curculionídeos foram amostrados semanalmente no período de março/2008 a fevereiro/2009, com a utilização de doze armadilhas tipo Moericke, confeccionadas com bandejas plásticas amarelas $(39 \mathrm{~cm}$ de comprimento x $29 \mathrm{~cm}$ de largura x $6 \mathrm{~cm}$ de profundidade). As armadilhas foram instaladas sob a copa das plantas ao nível do solo, distanciadas entre si por $22,5 \mathrm{~m} \mathrm{e}$ permaneceram ativas durante sete dias, sendo que cada uma foi preenchida com 1,5 $\mathrm{L}$ de água, $15 \mathrm{ml}$ de solução de formalina a $37 \%$, e algumas gotas de detergente neutro.

O material coletado foi transportado ao laboratório de Controle Biológico do INCAPER em Linhares, onde os curculionídeos foram separados dos demais grupos de insetos, e acondicionados em frascos contendo álcool 70\%. Posteriormente os exemplares foram encaminhados para o especialista Dr. Sergio Antonio Vanin do Museu de Zoologia da Universidade de São Paulo, SP, para identificação. Os exemplares encontram-se depositados no Museu de Zoologia da Universidade de São Paulo. 


\section{RESULTADOS E DISCUSSÃO}

Foi coletado um total de 952 exemplares de curculionídeos de onze espécies (Tabela 1), sendo que as mais frequentemente coletadas foram Parisoschoenus obesulus Casey 1922 (43,3\%), Andranthobius bondari (Hustache, 1940) (30,6\%) e Metamasius hemipterus (Linnaeus, 1758) (9,2\%). As demais representaram juntas, $17 \%$ do total coletado.

A espécie $P$. obesulus foi a mais abundante e ocorreu durante todo o período de levantamento. De acordo com Ferreira $e t$ al. (1997) este inseto é considerado praga severa para o coqueiro anão verde, pois as larvas provocam a queda dos frutos nos primeiros estágios de seu desenvolvimento; entretanto, estudos desenvolvidos em campo e laboratório mostraram que este inseto ataca somente frutos em processo de abortamento, não constituindo praga para o coqueiro (Moura \& Vilela, 1998; Moura et al., 2009).
Os picos populacionais de $P$. obesulus foram constatados nos meses de outubro e dezembro (Figura 1), no período da estação chuvosa na região; esta espécie foi relatada em C. nucifera em alguns estados do Nordeste (Bahia, Sergipe e Pernambuco) por Ferreira et al. (1998) e em São Paulo por Sánchez \& Nakano (2003).

A. bondari, segunda espécie mais coletada, também ocorreu durante todo período de levantamento, e já foi relatada associada a inflorescências masculinas de $C$. nucifera, $C$. coronata Mart. e em $C$. botryophora Mart. por Lima (1956), embora o autor não tenha relatado danos causados por esta espécie. Santos et al. (2003) coletaram 87 exemplares dessa espécie em palmeiras nativas da Amazônia, e Storti (1993) afirmaram que este gorgulho constitui-se um importante polinizador na palmeira buriti (Mauritia flexuosa Lin. Fil).

Tabela 1. Curculionidae (Coleoptera) coletados com armadilha de Moericke em cultura de coqueiro anão verde, no município de Linhares, ES, entre março de 2008 e fevereiro de 2009.

\begin{tabular}{|c|c|c|c|c|c|c|c|c|c|c|c|c|c|}
\hline Espécies & $\begin{array}{l}\stackrel{\infty}{\stackrel{\Xi}{\Xi}} \\
\stackrel{\Xi}{\Xi}\end{array}$ & 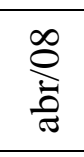 & 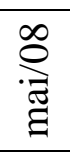 & 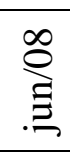 & $\stackrel{\infty}{\stackrel{\varrho}{\Xi}}$ & $\begin{array}{l}\infty \\
\stackrel{\infty}{\circ} \\
\stackrel{\varpi}{\infty}\end{array}$ & $\stackrel{\infty}{\stackrel{\infty}{d}}$ & $\stackrel{\infty}{\stackrel{\infty}{0}}$ & 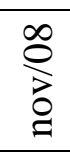 & 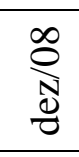 & 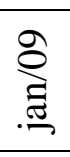 & 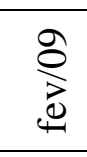 & $\stackrel{\widetilde{\sigma}}{0}$ \\
\hline Metamasius hemipterus & 33 & 8 & 4 & 1 & 0 & 2 & 1 & 1 & 5 & 28 & 2 & 3 & 88 \\
\hline Andranthobius bondari & 76 & 52 & 21 & 18 & 7 & 7 & 13 & 22 & 5 & 17 & 13 & 42 & 293 \\
\hline Parisoschoenus obesulus & 29 & 40 & 16 & 15 & 8 & 15 & 26 & 90 & 32 & 96 & 15 & 27 & 409 \\
\hline Baris sp. & 6 & 12 & 3 & 6 & 0 & 0 & 3 & 3 & 1 & 4 & 1 & 2 & 41 \\
\hline Apion sp. & 2 & 1 & 5 & 3 & 10 & 13 & 7 & 6 & 8 & 1 & 2 & 7 & 65 \\
\hline Sitophilus zeamais & 0 & 1 & 0 & 0 & 0 & 1 & 0 & 0 & 0 & 2 & 1 & 5 & 10 \\
\hline Conotrachelus sp. & 0 & 1 & 0 & 1 & 0 & 2 & 0 & 2 & 0 & 0 & 0 & 7 & 13 \\
\hline Auletobius sp. & 1 & 1 & 1 & 0 & 2 & 2 & 2 & 1 & 1 & 0 & 0 & 2 & 13 \\
\hline Sibinia sp. & 1 & 0 & 0 & 0 & 0 & 0 & 0 & 0 & 1 & 0 & 0 & 1 & 3 \\
\hline Anthonomus sp. & 0 & 0 & 0 & 0 & 0 & 0 & 0 & 0 & 0 & 0 & 2 & 1 & 3 \\
\hline Sibariops sp. & 0 & 0 & 0 & 0 & 0 & 3 & 2 & 1 & 1 & 2 & 2 & 3 & 14 \\
\hline Total & 148 & 116 & 50 & 44 & 27 & 45 & 54 & 126 & 54 & 150 & 38 & 100 & 952 \\
\hline
\end{tabular}




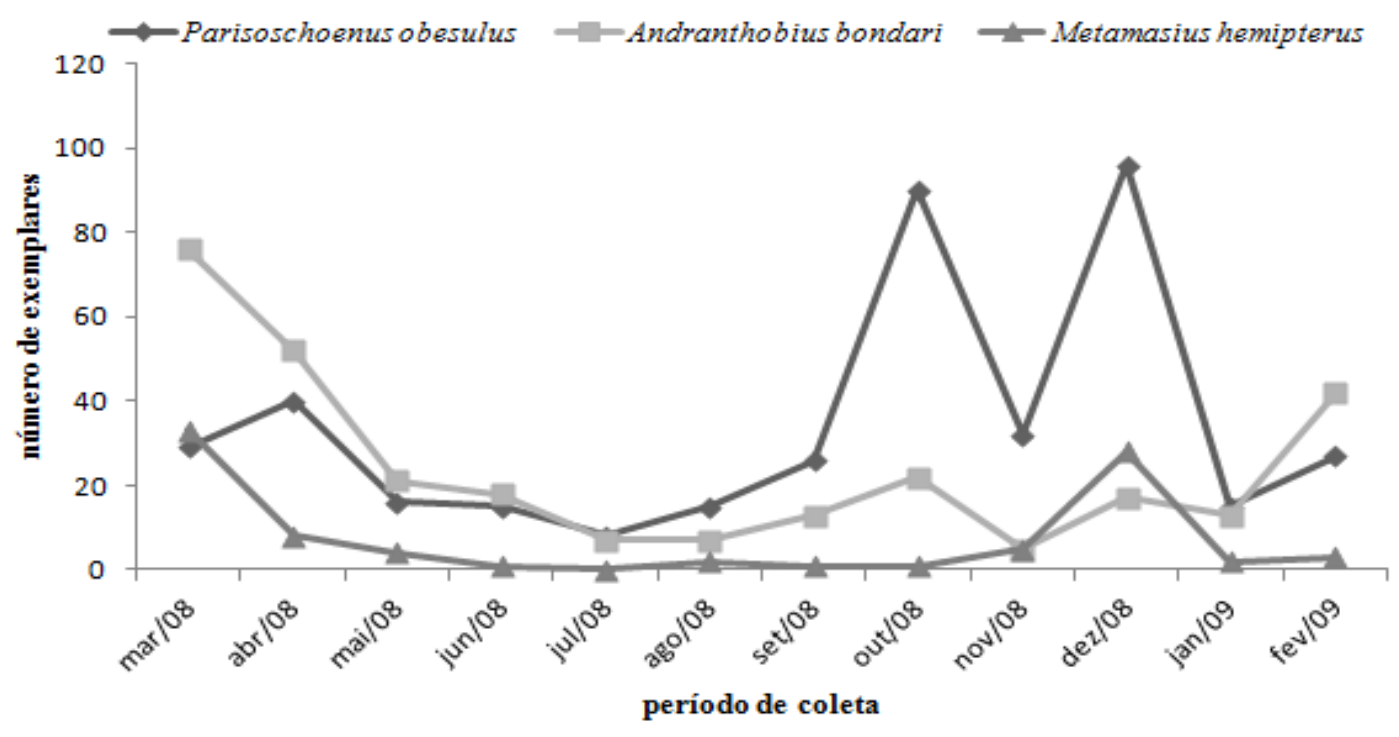

Figura 1. Flutuação populacional de Parisoschoenus obesulus Casey, 1922, Andranthobius bondari (Hustache, 1940), Metamasius hemipterus (L., 1758) (Coleoptera, Curculionidae), coletadas em cultivo de coqueiro anão verde, em Linhares, ES, entre março de 2008 e fevereiro de 2009.

A espécie M. hemipterus está associada a várias plantas hospedeiras como cana-de-açúcar, coqueiro, bananeira e gramíneas, e foi registrada em diversos estados brasileiros, inclusive no Espírito Santo (Silva \& Martins-Silva, 1991). Estes mesmos autores relataram ser a espécie, vetora da "doença do anel vermelho" causada pelo nematóide Rhadinaphelenchus cocophilus (Cobb, 1919), entretanto, a sua ocorrência neste levantamento, não indica, necessariamente, que o mesmo constitui ameaça no coqueiral avaliado, uma vez que, próximo à área de coqueiro estudada, havia uma cultura de bananeira e de cana-deaçúcar. Também não foram observadas plantas de coqueiro com sintomas da "doença do anel vermelho". Todavia, vale salientar que a presença de culturas hospedeiras adjacentes, pode favorecer sua dispersão para o coqueiral, favorecendo sua disseminação no cultivo.
Apesar de o coqueiro anão verde ser atacado por várias espécies de curculionídeos, as demais espécies capturadas não estão registradas na literatura como hospedeiras para C. nucifera. Provavelmente, sua ocorrência pode estar relacionada com a presença de plantas invasoras que eram comuns na cultura durante o período de estudo, as quais podem fornecer alimento a esses insetos, fato também relatado por Mexzón \& Chinchilla (1998) que encontraram espécies de Curculionidae não pragas em cultivo de dendezeiro na Costa Rica visitando plantas invasoras presentes nas entrelinhas do cultivo.

\section{AGRADECIMENTOS}

Os autores agradecem ao pesquisador Dr. Sergio Antonio Vanin do Museu de Zoologia da Universidade de São Paulo, SP, 
pela atenção e identificação das espécies de curculinídeos.

\section{REFERÊNCIAS BIBLIOGRÁFICAS}

LIMA, A. M. C. 1956. Insetos do Brasil. Coleópteros, 4. parte. Rio de Janeiro: Escola Nacional de Agronomia. v.10, 373p. (Série Didática n.12).

FAO 2011. World Production. Disponível em: <www.faostat.org.br>. Acesso em: 10 set.

FERREIRA, J. M. S.; LIMA, M. F.; SANTANA, D. L. Q.; MOURA, J. I. L. 1998. Pragas do coqueiro, p. 81-118. In: SOBRINHO, R. B.; CARDOSO, J. E.; FREIRE, F. C. O. (eds.), Pragas de fruteiras tropicais de importância agroindustrial. Brasília: Embrapa-SPI, Fortaleza, Embrapa-CNPAT, 209p.

FERREIRA, J. M. S.; WARWICK, D. R. N., SIQUEIRA, L. A. 1997. A cultura do coqueiro no Brasil. Brasília: EMBRAPA-SPI; Aracaju : EMBRAPACPATC, $2^{\mathrm{a}}$ ed., 297p.

MARTINS, C. R.; JESUS JÚNIOR, L. A. 2011. Evolução da produção de coco no Brasil e o comércio internacional: panorama 2010. Aracaju : Embrapa Tabuleiros Costeiros, 2011. (Documentos / Embrapa Tabuleiros Costeiros, 164). 28 p.

MEXZÓN, R. G.; M. CHINCHILLA , C. M. I. 1998. Plant species attractive to beneficial entomofauna in oil palm (Elaeis guineensis Jacq.) plantations in Costa Rica. Oil Palm Papers, San José, n. 19, p. 1-22. Disponível em: <http://www.asd-cr.com/paginas/english /articulos/bol19-1en. html>. Acesso em: 16 set. 2010.

MOURA, J. I. L.; FERREIRA, J. M. S.; SGRILLO, R. B.; VALLE, R. R.; ALMEIDA, A. A. F.; CIVIDANES, F. J.; DELABIE, J. H. C. 2009. Parisoschoenus obesulus Casey (Coleoptera: Curculionidae) não é praga de frutos novos do coqueiro. Neotropical Entomology, Londrina, v. 38, n. 2, p. 251-253.

MOURA, J. I. L.; VILELA E. F. 1998. Pragas do coqueiro e dendezeiro. 2 ed. Viçosa: Aprenda Fácil. 124p.

SÁNCHEZ, S.; NAKANO, O. 2003. Presença de Parisoschoenus obesulus Casey (Coleoptera: Curculionidae) na Cultura do Coqueiro no Estado de São Paulo, Brasil. Entomotropica, Maracay, v. 18, n. 1, p. 77-78.

SANTOS, G. B.; MARQUES, M. I.; ADIS, J.; DE MUSIS, C. R. 2003. Artrópodos associados à copa de Attalea phalerata Mart. (Arecaceae), na região do Pantanal de Poconé, Mato Grosso, Brasil. Revista Brasileira de Entomologia, Curitiba, v. 47, n. 2, p. 211- 224.

SILVA, H. M.; MARTINS-SILVA, H. 1991. Metamazius sp. (Coleoptera: Curculionidae) vetor do Rhadinaphelenchus cocophilus agente causador do anel vermelho do dendezeiro. Unidade de Execução de Pesquisa de Âmbito Estadual de Belém, EMBRAPA, n.17, 4p.

STORI, E. F. 1993. Biologia floral de Mauritia flexuosa Lin. Fil, na região Manaus, AM, Brasil. Acta Amazônica, Manaus, v. 23, n. 4, p. 371-381. 